\title{
Psychiatric day hospital discharge summaries
}

\author{
An evaluation by general practitioners of a new model
}

\author{
Kim Shamash, Associate Research Fellow, Archway 15, Whittington Hospital, \\ Highgate Hill, London N19 5NF (correspondence); GEORGE IKKOs, Senior Registrar, \\ Department of Psychological Medicine, University College Hospital; SHAMIL \\ Wanigaratne, Senior Clinical Psychologist, James Pringle House, Middlesex \\ Hospital, London W1; and MAURICE GreENBERG, Consultant Psychiatrist, Jules \\ Thorn Day Hospital, St Pancras Hospital, London NW1 and University College \\ Hospital, Gower Street, London WC1
}

With the increasing emphasis on community care (Turner, 1986) the psychiatric day hospital has been seen as an alternative (Tantam, 1985) or as a complement (Tyrer, 1985) to in-patient care. Associated with this is the development of new styles of working with patients such as the 'multidisciplinary team' with those looking after individual patients being referred to as 'key workers' (Watts \& Bennett, 1983). In our day hospital the keyworker has considerable responsibility for history-taking, organising a treatment programme and communicating with other professionals, including writing the discharge summary.

The Psychiatric Discharge Summary (PDS) provides a valuable record of communication about assessment and treatment but its purpose, and hence form and content, depends on the recipient's needs. Writing a PDS is therefore a skilled task and we wanted to know whether this task could be effectively delegated to professionals with no previous training.

An earlier survey of local GPs found that $97 \%$ read all or most of the summaries and $90 \%$ read each summary thoroughly or almost completely (Orrell \& Greenberg, 1986). Usefulness of the summary was found to be significantly related to the inclusion of details regarding diagnosis, prognosis and follow-up. In addition GPs felt that information about followup and what the patient and relatives had been told was important but often omitted. Many also said that they would prefer the summary in a concise and easily stored form.

Inspection of the day hospital summaries showed that they were often several pages long and written in a discursive style more appropriate for internal use than for GP consumption. It was frequently noted that essential information about previous treatment was missing. This was so whether or not the keyworker was medically qualified.

\section{The study}

A new model of summary was proposed taking account of suggestions highlighted by the previous study. The main change was the creation of a separate section at the head of the summary for information relating to diagnosis, medication on discharge and plans for future management giving these details a more prominent position. There was an additional heading for information given to the patient and relatives and we suggested a maximum length of two sides of A4 paper.

By means of a series of seminars and individual discussions one of the authors (GI) taught members of the multidisciplinary team how to write summaries. One result of these discussions was that the psychiatric registrar retained responsibility for the 'medical' aspects and shared overall responsibility for the summary with the keyworker.

Once the new model had been established for a year, six new model summaries (NM) were randomly selected and compared to six summaries written before the teaching exercise had occurred (OM). The numbers were chosen to ensure a balance between a representative sample and the optimal number of times each summary would be read and summaries were matched for age, sex, diagnosis and length of stay of the patient. The number of words and appropriate headings were counted and an evaluation form was designed to obtain ratings for usefulness of content and layout with a section for open-ended comments. GPs from a wide area were then invited to take part.

Each GP who agreed was sent a package containing one summary from each group, with a questionnaire attached to each one. They were asked to read the summaries in the order in which they were presented and to complete the corresponding 
questionnaire before proceeding to the next summary.

To control for order effects the sequence was randomised and to ensure that all summaries were read an equal number of times the design adopted a serial method of summary allocation to each condition, the GPs being randomly allocated to each condition. The mean rating of the groups for each item was compared using the Wilcoxon matched-pairs signedranks test.

\section{Findings}

Of the 50 GPs who agreed to take part, $42(84 \%)$ returned completed questionnaires. The proportions in single-handed and group practices were the same as in the district as a whole. The two groups in the summaries were comparable in terms of length of stay and diagnosis of patient but NM were briefer with a greater number of appropriate headings.

There were significant differences in favour of NM in the ratings for usefulness on the following five items: family history, past drug treatment, results of special investigations, information given to relatives and possible future development of the disorder. They were also significantly preferred for ease of handling, ease of reading and appropriateness of length. The overall scores for NM were significantly higher than those for OM. In 15 of the 25 items there was no significant difference and there was a significant difference in favour of $\mathrm{OM}$ for the item on responsibility for future management.

The comments section indicated that GPs were fairly satisfied with both models. They liked the essay-like style of OM but sometimes found them long and repetitive. Conversely they found NM wellsummarised and easy to assimilate but with a cramped and impersonal style.

\section{Comment}

These results demonstrate that it is possible to improve summaries since in most areas NM were either as good as or better than OM and that non-medical colleagues with medical supervision can be taught to write PDS. It may be that the high response rate is attributable to the criteria by which GPs were selected. However, since the proportion of responders in single-handed to group practices is the same as that in the district as a whole, we believe that this was a representative sample.

We were surprised at the item on Responsibility for Future Management but detailed inspection of the summaries suggested the following explanation. Both groups of summaries had maintained the anonymity of the patient, staff and unit but the discursive nature of OM meant that there was usually reference to follow-up arrangements in the text whereas in NM there was merely an outline of follow-up plans at the head of the summary which was sometimes missed by the GP. Thus information about who is to be responsible for follow-up needs to be stated more definitely.

Although we have shown that it is possible to teach non-medical staff to write PDS which GPs prefer, we were disappointed that the improvements gained were at the expense of a narrative quality which had previously been valued. We may have underestimated the skill that is required to produce not just a concise document containing a number of useful facts, but also one that is interesting to read. Whether this skill can also be taught has not been shown but it is likely to be affected by individual factors as well as educational background and professional training.

It should be emphasised that although the initial teaching input was minimal, the standard of summaries was maintained by continued supervision from medical staff. This collaborative process allowed recognition of the key worker's special knowledge of the individual and helped in the approach to assessing and communicating about patients. It enabled medical staff to understand the experiences of other staff with patients and perhaps to offer a skill in an environment where the junior doctor can easily feel deskilled. It therefore seems valuable in increasing staff cohesion (Watts \& Bennett, 1983).

We would encourage the adoption of this collaborative process in psychiatric day hospitals but with greater emphasis being given to responsibility for future management. However such an approach may not be suitable for acute in-patient units where the doctor is in effect the keyworker.

\section{Acknowledgements}

We wish to thank all the staff of Jules Thorn Day Hospital for their enthusiasm and patience, Dr Chris Barker for his advice and all the GPs who so generously gave of their valuable time to take part.

\section{References}

OrRell, M. W. \& Greenberg, M. (1986) What makes psychiatric summaries useful to general practitioners? Bulletin of the Royal College of Psychiatrists, 10, 104-107.

TANTAM, D. (1985) Alternatives to psychiatric hospitalisation. British Journal of Psychiatry, 146, 1-4.

TURNER, T. (1986) Community care. British Journal of Psychiatry, 152, 1-3.

TYRER, P. (1985) The hive system: A model for a psychiatric service. British Journal of Psychiatry, 146, 571-575.

WATTS, F. \& BENNETT, D. (1983) Management of the staff team. In Theory and Practice of Psychiatric Rehabilitation (eds. G. N. Watts \& D. W. Bennett). Chichester: John Wiley. 